\title{
Experimental and theoretical studies on the anti-amyloidogenic and destabilizing effects of pyrogallol against Human insulin protein
}

Parastoo Shouhani ${ }^{1}$, Seifollah Bahramikia*2, Seyed Hesam Hejazi ${ }^{2}$

${ }^{1}$ Department of Biology, MSc of Biology, Lorestan University, Khorramabad, Iran

${ }^{2}$ Department of Biology, Faculty of Basic Sciences, Lorestan University, Khorramabad, Iran

* Corresponding author:

Seifollah Bahramikia Tel: +989166614082 Fax: +986633120100

E-mail address: bahramikia.s@lu.ac.ir

ABSTRACT: One of the major problems caused by repeated subcutaneous insulin injections in diabetic patients is insulin amyloidosis. Understanding the molecular mechanism of amyloid fibril formation of insulin and finding effective compounds to its inhibit or eliminate is very important and extensive research has been done on it. In this study, the anti-amyloidogenic and destabilizing effects of the pyrogallol, as a phenolic compound, on human insulin protein were investigated by CR absorbance, ThT and ANS fluorescence, FTIR spectroscopy and atomic force microscopy. According to the obtained results, the formation of amyloid fibrils at $\mathrm{pH} 2.0$ and $50{ }^{\circ} \mathrm{C}$ was confirmed by $\mathrm{CR}$, ThT, ANS, FTIR assays. Microscopic images also showed the twisted and long structures of amyloid fibrils. Simultaneous incubation of the protein with pyrogallol at different concentrations reduced the intensities of CR, ThT and ANS in a dose-dependent manner and no trace of fibrillar structures was observed in the microscopic images. FTIR spectroscopy also showed that the position of amide I band in the spectrum of samples containing pyrogallol was shifted. Based on the findings in this study, it can be concluded that pyrogallol can be effective in preventing and suppressing human insulin amyloid fibrils. 


\section{KEYWORDS}

Human Insulin, Amyloid Fibrils, Protein aggregation, Amorphous aggregates, Aromatic Compounds, Polyphenol, Pyrogallol

\section{Introduction}

Diseases related to protein misfolding are disorders caused by various factors such as genetic mutation, heat shock and malfunction of monitoring systems. As a result of these processes, the correct process of protein folding is disrupted and leads to its opening and exposure to hydrophobic surfaces. Following the change in protein structure, incorrect interactions occur between adjacent protein molecules, and eventually, very orderly and insoluble structures called amyloid fibrils are formed [1-4]. Many amyloidogenic proteins such as amyloid $\beta, \alpha$-synuclein, islet amyloid polypeptide, and human lysozyme have been associated with protein misfolding diseases [5]. Extracellular aggregation and deposition of amyloid fibrils lead to cell death and in many cases cytotoxicity [4].

Insulin hormone is a small globular protein with 51 amino acid residues [6]. This hormone plays an important role in regulating glucose metabolism and is used in the treatment of diabetes [7]. Insulin fibrillation in the body may occur after continuous and subcutaneous injection in diabetic patients and lead to local deposition of insulin amyloid fibrils at the injection site [8,9]. On the other hand, fibrillation is also one of the problems that occur during the production and storage of insulin as well as the agitation of insulin solutions during transport [7,10]. In vitro factors such as temperature, $\mathrm{pH}$, protein concentration, ionic strength, the presence of organic solvents and denaturants such as urea and guanidine chloride are effective in changing the structure and formation of insulin amyloid fibrils $[11,12]$. 
In recent years, finding a strategy for the treatment of amyloid diseases has been considered by many researchers. Important treatment strategies proven in most of these studies are to prevent the formation of amyloid fibrils and their disaggregation. It has been shown that small organic molecules, including polyphenols, due to having aromatic rings, possess the ability to interact and bind to proteins and show significant anti-amyloidogenic effects. In addition, the antioxidant properties of polyphenols have made these compounds very effective in clearing free radicals and thus reducing cancer, as well as in the treatment of chronic and degenerative diseases [13-15]. Reduction of amyloid-induced cell death and inhibition of self-association of amyloidogenic proteins in vitro are other beneficial functions of polyphenols [16].

In the structure of polyphenols, there is at least one aromatic ring with hydroxyl groups attached to it. Due to the number and location of these phenolic rings, as well as the presence of other constituent elements, various structures of polyphenols have emerged [17,18]. Phenolic compounds are found in large quantities mainly in natural foods such as vegetables, fruits, seeds, nuts, as well as in flowers. Due to their natural origin and presence in the human diet, they are given more attention than other inhibitory molecules [19,20].

1,2,3-Trihydroxybenzene, commonly known as pyrogallol, is a phenolic compound like other natural polyphenols that is present in human food sources, including fruits and vegetables, and a variety of edible and medicinal plants [20]. Pyrogallol, through hydroxyl groups attached to its aromatic ring, has a high tendency to form non-covalent interactions such as hydrogen bonds and hydrophobic with a variety of biological macromolecules, including proteins [21]. In addition, it has been proven that pyrogallol possesses a high potential in the elimination of free radicals [22].

In this study, from the phenolic compound of pyrogallol was used with aim to investigate its antiamyloidogenic and destabilizing effects on the process of fibrillation and defibrillation of human insulin protein in vitro by several techniques such as CR absorbance, ThT and ANS fluorescence, FTIR spectroscopy and atomic force microscopy. 


\section{Results}

\subsection{Investigation of the effects of Py on the process of $\mathrm{HI}$ fibrillation}

\subsubsection{Effect of Py on the kinetics of HI fibril formation}

To investigate the effect of Py on the kinetics of HI fibril formation, ThT fluorescence of HI incubated in the absence and in the presence of different concentrations of Py was measured at different times. The sigmoid curve obtained in this study showed that HI fibrillation follows the nucleation-dependent elongation mechanism [23].

According to the results presented in Fig. 1 and Tab. 1; the lag phase time of HI incubated in the absence of Py is about 47.29 hours, after which the intensity of ThT fluorescence increases strongly and enters the fibril growth phase. Incubation of insulin in the presence of different concentrations of Py resulted in a reduction of the final ThT fluorescence intensity (increasing the inhibition percentage) in a dose-dependent manner (Tab. 1). The high dose of Py (HI+Py (1:5)), in addition to having the highest effect on reducing fibril formation, was somewhat more effective in prolonging

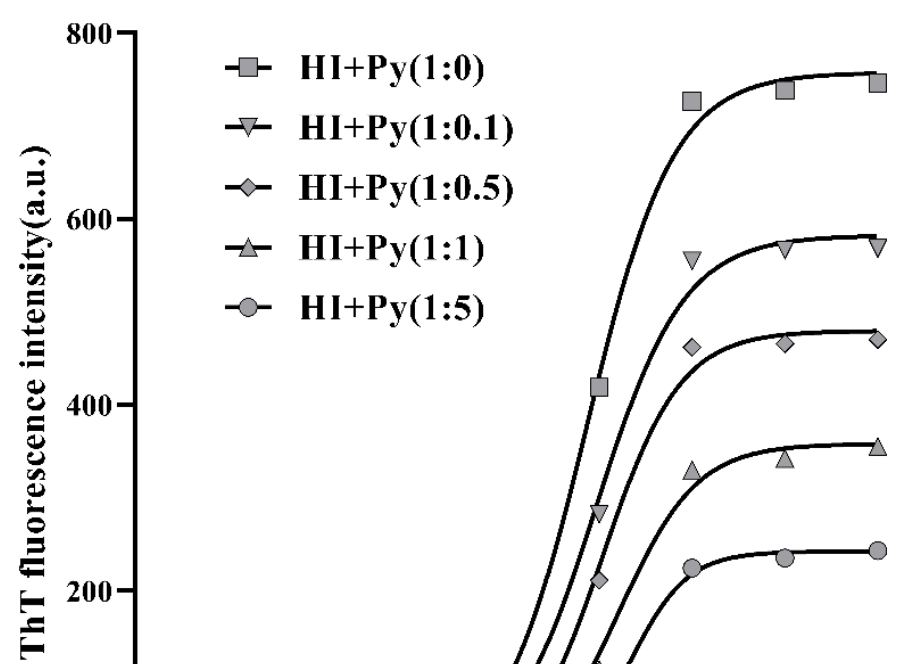

Fig. 1. Effects of pyrogallol on the fibrillation kinetics of human insulin. ThT fluorescence intensity of human insulin was recorded at $485 \mathrm{~nm}$ in the absence and in the presence of different concentrations of pyrogallol at $\mathrm{pH} 2.0$ and $50^{\circ} \mathrm{C}$ at different times. 
the lag phase time of this process than other concentrations. In the presence of Py, no effect was observed on reducing the rate of the growth of fibril.

Table 1. Effects of different concentrations of pyrogallol on the kinetic parameters of human insulin fibril formation process.

\begin{tabular}{lll}
\hline & $\begin{array}{l}\text { Lag time } \\
\text { (h) }\end{array}$ & $\begin{array}{l}\text { Inhibition } \\
\text { (\%) }\end{array}$ \\
\hline HI+Py (1:0) & 47.29 & - \\
\hline HI+Py (1:0.1) & 48.33 & 23.79 \\
\hline HI+Py (1:0.5) & 50.64 & 36.97 \\
\hline HI+Py (1:1) & 52.70 & 52.40 \\
\hline HI+Py (1:5) & 56.28 & 67.44 \\
\hline
\end{tabular}

\subsubsection{CR, ThT, ANS and FTIR spectroscopy}

According to the results presented in Fig. 2A; the CR absorbance intensity of incubated HI in the absence of Py increased and its maximum optical absorption has red-shifted from the wavelength of $490 \mathrm{~nm}$ to the wavelength of $500 \mathrm{~nm}$. When insulin was incubated in the presence of different concentrations of Py, a decrease in the CR absorbance intensity was observed, dose-dependently. In the ratios of 1:1 and 1:5 $\mathrm{HI}$ to Py, in addition to a significant decrease in the absorbance intensity, a blue shift (shift maximum absorption from $500 \mathrm{~nm}$ to $490 \mathrm{~nm}$ ) was also observed. Gallic acid (Ga) is a polyphenol composed of trihydroxy benzene (Py) and a carboxyl group. In order to compare the inhibitory effect of Ga with Py due to the structural similarity of these two compounds and to understand whether the carboxyl group has an effect on inhibiting the fibrillation process, a ratio of 1:5 HI to Ga was prepared and its effect on the fibrillation process was investigated. As shown in Fig. 
2A; it was observed that Ga reduced the CR absorbance intensity of incubated HI by approximately as much as the ratio of 1:5 $\mathrm{HI}$ to Py.

According to the results presented in Fig. 2B; the ThT fluorescence intensity of incubated HI in the absence of Py at $485 \mathrm{~nm}$ compared to pre-incubation HI (native HI) increased strongly. This result confirms the results of the CR assay. In the presence of different concentrations of Py, the ThT fluorescence intensity of incubated HI decreased in a dose-dependent manner. In addition, The ThT fluorescence intensity of HI samples incubated with Ga at the same concentration decreased and confirms the CR assay results.
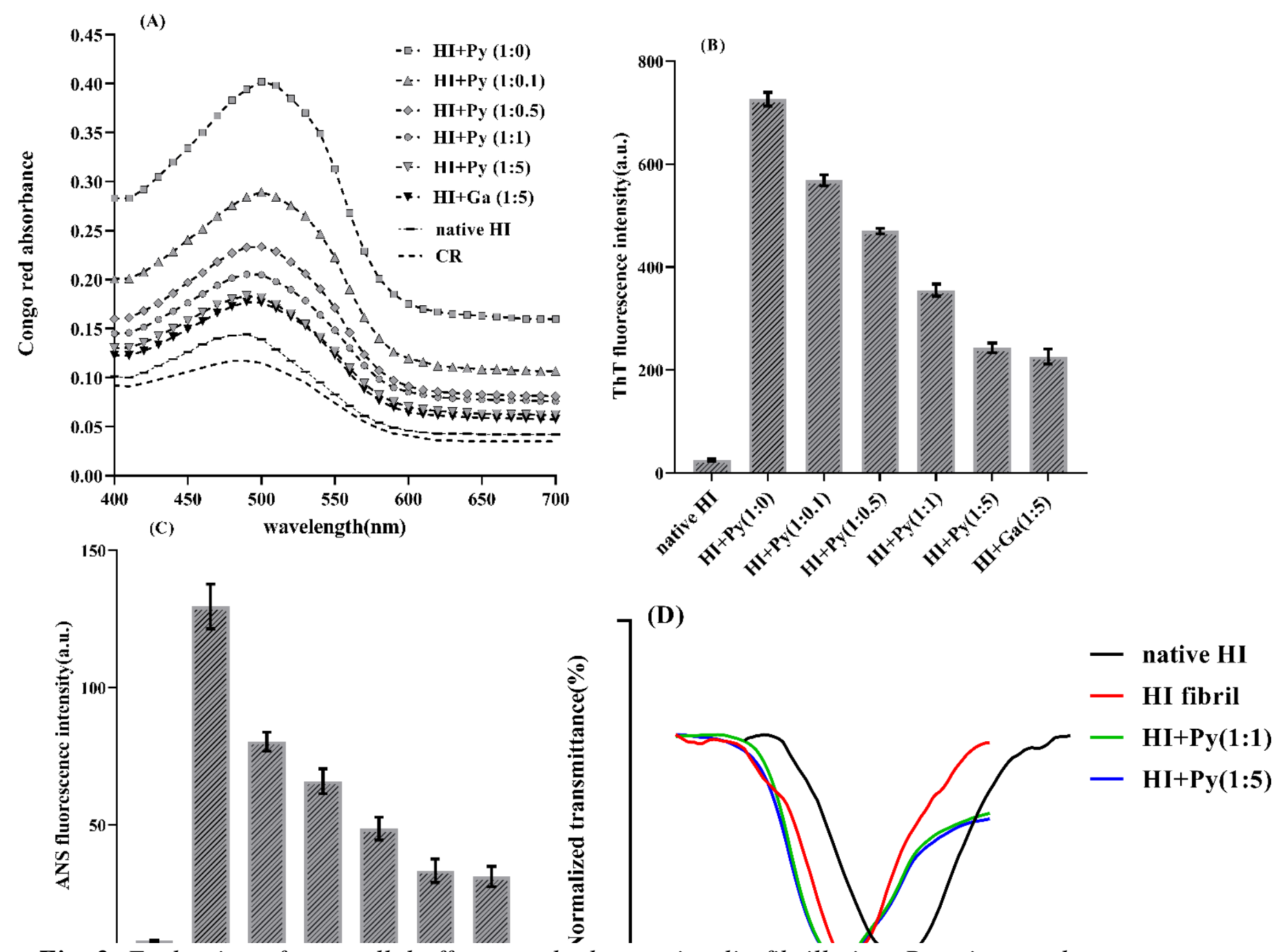

Fig. 2. Evaluation of pyrogallol effects on the human insulin fibrillation. Protein samples were tested in the absence and in the presence of different concentrations of pyrogallol after the incubation at $\mathrm{pH} 2.0$ and at $50{ }^{\circ} \mathrm{C}$. (A) CR test (B) ThT fluorescence test (C) ANS fluorescence test and (D) FTIR spectroscopic test. In statistical analysis (B,C), pre-incubation human insulin (native human insulin) was considered as a control to compare other groups with it. In this comparison, $P<0.0001$ was for all groups. The results are the average of three independent experiments. 
According to the results presented in Fig. 2C; the ANS fluorescence intensity of pre-incubation $\mathrm{HI}$ is low due to the hydrophobic regions buried inside the protein structure and the lack of ANS access to them. When $\mathrm{HI}$ is incubated under acidic conditions at $50{ }^{\circ} \mathrm{C}$ the ANS fluorescence intensity was significantly increased. In the investigating of samples containing Py, it was observed that with increasing the concentration of this compound, its effectiveness in reducing the ANS fluorescence intensity of incubated HI increases. The same decrease in ANS fluorescence intensity of incubated HI by Py and Ga also indicates a similar effect of these two compounds in reducing exposure of hydrophobic regions on the protein surface.

According to the results presented in Fig. 2D; The FTIR spectrum of pre-incubation HI showed an amide I band in region $1655 \mathrm{~cm}^{-1}$. After incubation, displacement was observed in the amide I band position and the FTIR spectrum of HI (HI fibril) showed the amide I band in the $1630 \mathrm{~cm}^{-1}$ region. According to previous reports [24] the range $1653-1660 \mathrm{~cm}^{-1}$ is attributed to the $\alpha$-helix conformation and the range $1615-1643 \mathrm{~cm}^{-1}$ is attributed to the $\beta$-sheet conformation. It was also reported that the $\beta$ structure of amyloid fibrils has been observed in the range of $1628-1632 \mathrm{~cm}^{-1}$. Therefore, FTIR measurement confirms the change in HI conformation from $\alpha$-helix to $\beta$-sheet and thus the presence of fibrillar structure in the sample after incubation. The FTIR spectrum of HI+Py in ratios of 1:1 and 1:5 showed the position of amide $\mathrm{I}$ band in region $1626 \mathrm{~cm}^{-1}$ which is in the range of $\beta$ structure of amorphous aggregates $\left(1624-1630 \mathrm{~cm}^{-1}\right)$ [24].

\subsection{Investigation of destabilizing effects of $\mathrm{HI}$ amyloid fibrils by $\mathrm{Py}$}

Pre-formed HI fibrils were incubated in the absence and in the presence of different concentrations of Py for 3 days at $37{ }^{\circ} \mathrm{C}$ and then their structural changes were evaluated by CR, ThT, ANS and FTIR spectroscopy.

According to the results presented in Fig. 3A and Fig. 3B; The CR absorbance and ThT intensity of Py-free fibrillar samples increased. In contrast, samples containing Py showed a reduction in the 
CR absorbance and ThT intensity, dose-dependently. In addition, in the study of CR absorbance, the ratios of 1:1 and 1:5 $\mathrm{HI}$ fibril to Py showed a blue shift (shift maximum absorption from $500 \mathrm{~nm}$ to $490 \mathrm{~nm})$.

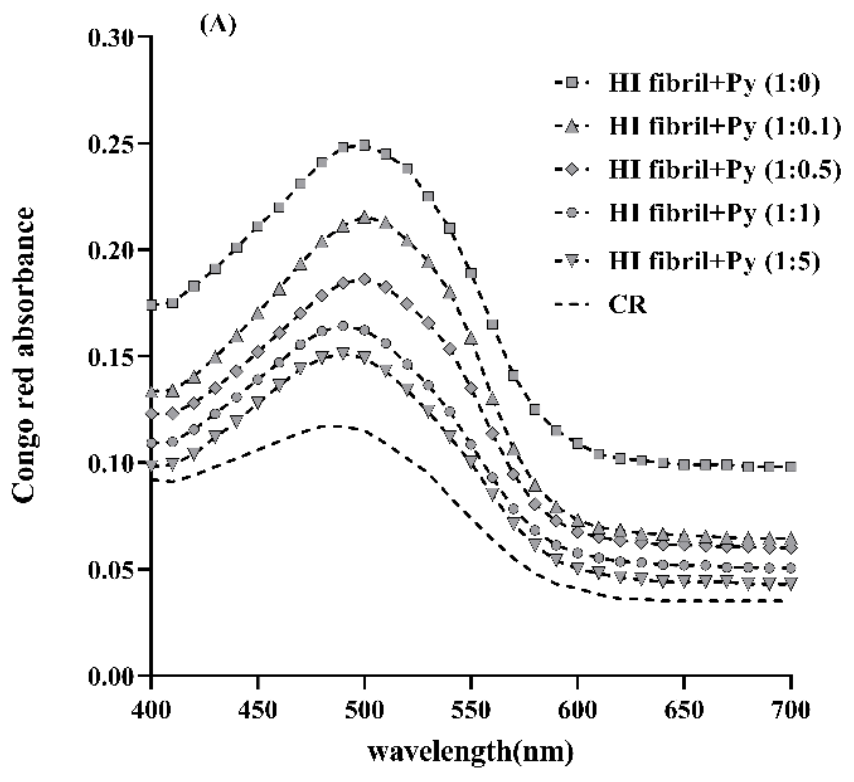

(B)
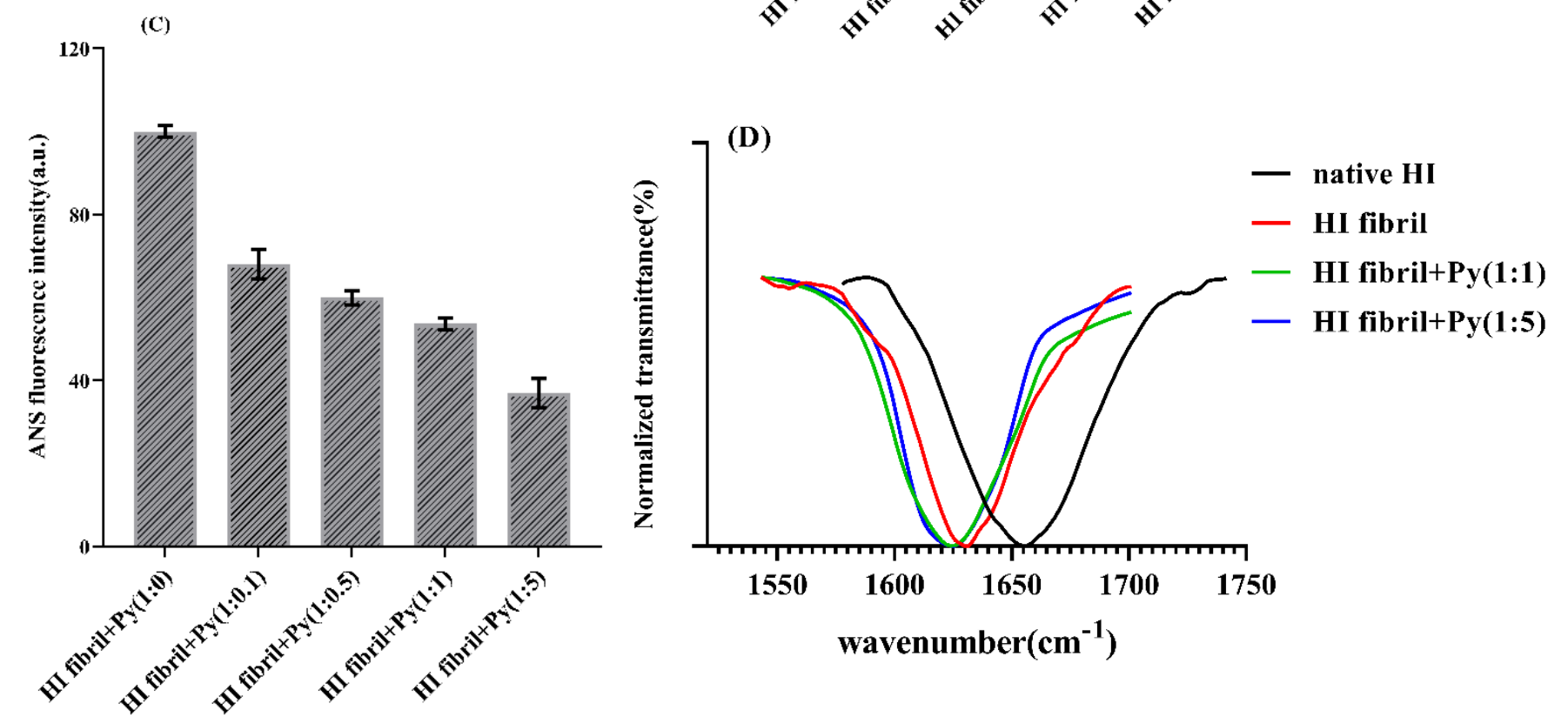

Fig. 3. Evaluation of pyrogallol effects on the human insulin defibrillation. Samples containing pre-formed human insulin fibrils were tested in the absence and in the presence of different concentrations of pyrogallol after incubation for 3 days at $37^{\circ} C$. (A) CR test (B) ThT fluorescence test (C) ANS fluorescence test and (D) FTIR spectroscopic test. In statistical analysis (B,C), human insulin fibril in the absence of pyrogallol was considered as a control to compare other groups with it. In this comparison, $P<0.0001$ was for all groups. The results are the average of three independent experiments. 
According to the results presented in Fig. 3C; in the investigating of the surface hydrophobicity of pre-formed HI fibrils by ANS fluorescence, it was found that all Py concentrations were effective in reducing the ANS intensity of fibrils and this effect was in a dose-dependent manner.

According to the results presented in Fig. 3D; Compared with the FTIR spectrum of HI fibril which showed the amide I band in the $1630 \mathrm{~cm}^{-1}$ region, the FTIR spectrum of the HI fibril+Py in the ratios of 1:1 and 1:5 showed the position of amide I band in region $1624 \mathrm{~cm}^{-1}$ which is in the range of $\beta$ structure of amorphous aggregates $\left(1624-1630 \mathrm{~cm}^{-1}\right)[24]$.

\subsection{AFM scanning}

AFM was used to investigate the effects of Py on the structural morphology of $\mathrm{HI}$ in fibrillation and defibrillation processes.

According to the results presented in Fig. 4A; after exposure to acidic $\mathrm{pH}$ and incubation at $50{ }^{\circ} \mathrm{C}$, twisted and long amyloid fibrils were observed in Py-free samples, however, as shown in Fig. 4B, in the presence of Py, no fibrillar structure was observed and the images showed granular structures instead of fibrillar morphology. It is possible that Py redirects the protein fibril formation pathway toward the creation of amorphous aggregation because it is observed that the amorphous aggregation in the electron microscope images has a granular appearance [2]. These results and the data of other tests performed in this study confirm the anti-amyloidogenic properties of Py.

As shown in Fig. 4C, Py also successfully acted in the elimination of amyloid fibrils and leads to a change in the fibrillar structure of the protein and probably the creation of amorphous aggregation. 

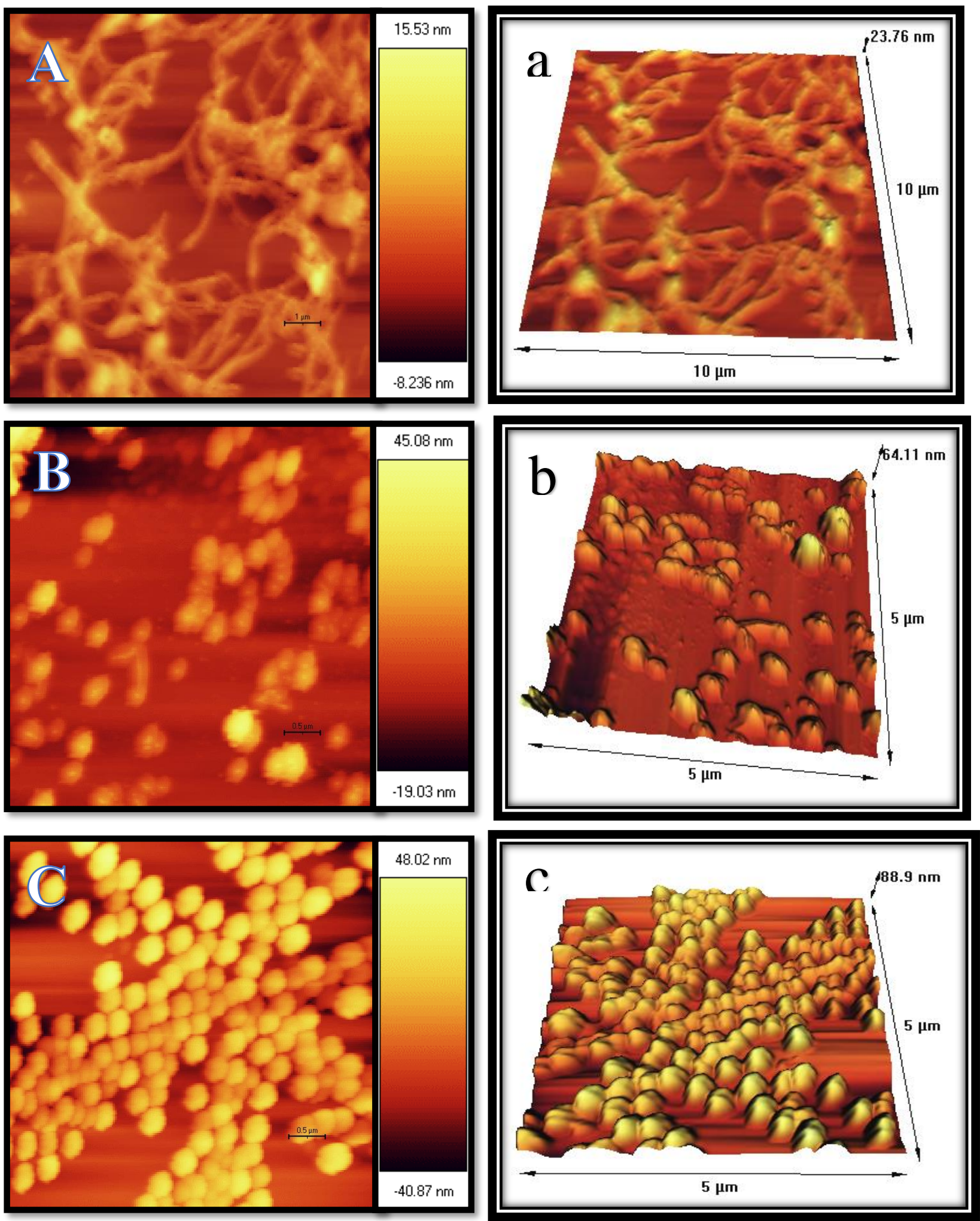

Fig. 4. Evaluation of pyrogallol effects on the human insulin morphology using AFM. (A) $2 D$ image and (a) $3 D$ image of pyrogallol-free human insulin fibrils in the fibrillation process (after incubation at $\mathrm{pH} 2.0$ and $50^{\circ} \mathrm{C}$ ). (B) $2 \mathrm{D}$ image and (b) $3 \mathrm{D}$ image of human insulin in the presence of pyrogallol $(\mathrm{HI}+\mathrm{Py}(1: 5))$ in the fibrillation process (after incubation at $\mathrm{pH} 2.0$ and $\left.50^{\circ} \mathrm{C}\right) .(\mathrm{C})$ $2 D$ image and (c) 3D image of pre-formed human insulin fibrils In the presence of pyrogallol (HI fibril $+P y(1: 5))$ in defibrillation process (after incubation at $37^{\circ} \mathrm{C}$ for 3 days). 


\subsection{Docking studies}

AutoDock software was used to better understand the interactions between HI and Py as well as to determine possible connection locations.

According to the results presented in Fig. 5; the amino acid residues of Gly1A, Ile2A, Val3A, Tyr19A, Tyr26B, and Thr27B interact with the ligand through hydrophobic interactions, and Thr27B also forms a hydrogen bond with the ligand (Length of hydrogen bond $2.93 \AA$ ).

Since the GFFYTPKT (Residues 23-30) peptide fragment of the insulin B chain helps to create aggregation [25], Py reacted with the two amino acid residues in this fragment (Tyr26B and Thr27B), whereby It can cause disruption in the process of fibril formation. Despite the lack of interaction of Py with the LVEALYL (Residues 11-17) peptide fragment of the insulin B chain which has a very high tendency to form amyloid $[11,26]$ it was shown that Py has a significant effect in preventing the formation of HI amyloid aggregation. In the study of molecular docking of ascorbic acid [27] and peptides P4 and P5 [28] also no interaction with this amyloidogenic fragment of HI was observed and the researchers concluded that the reaction of other amino acid residues with inhibitors leads to the prevention of their participation in the interactions that cause the creation of amyloid structures.
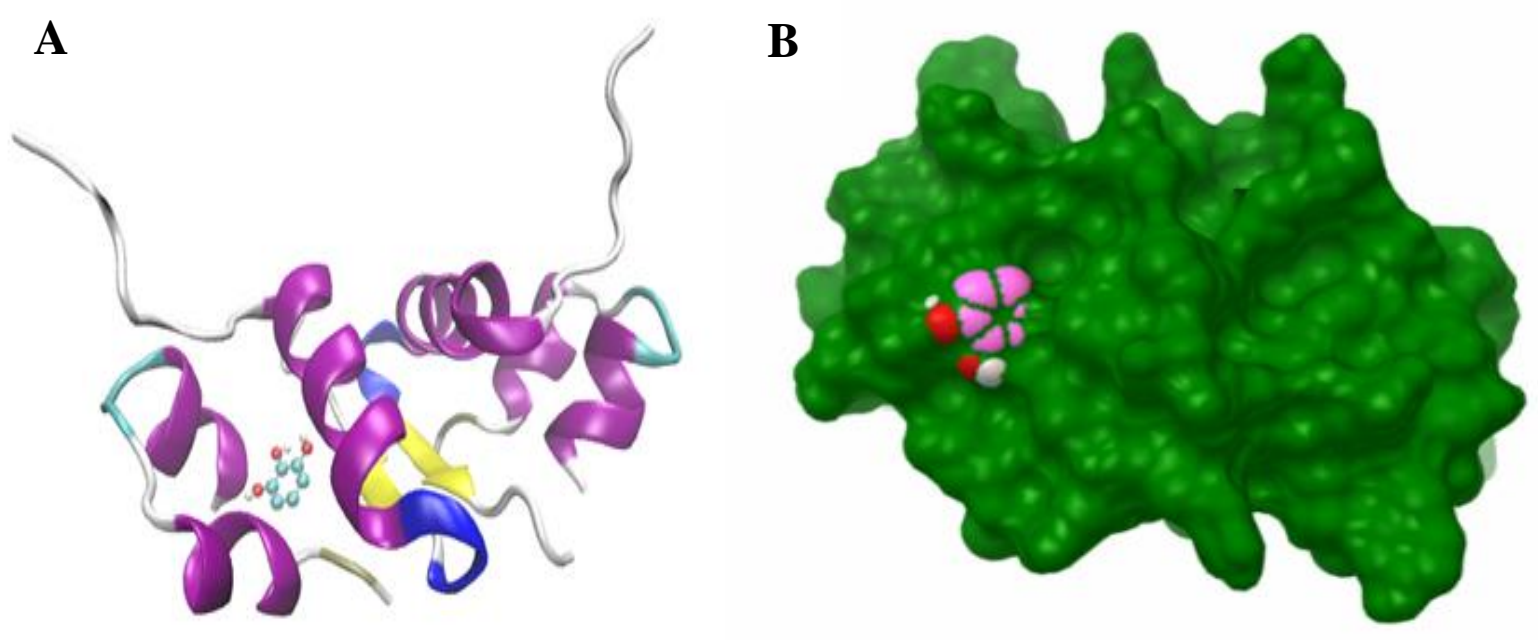


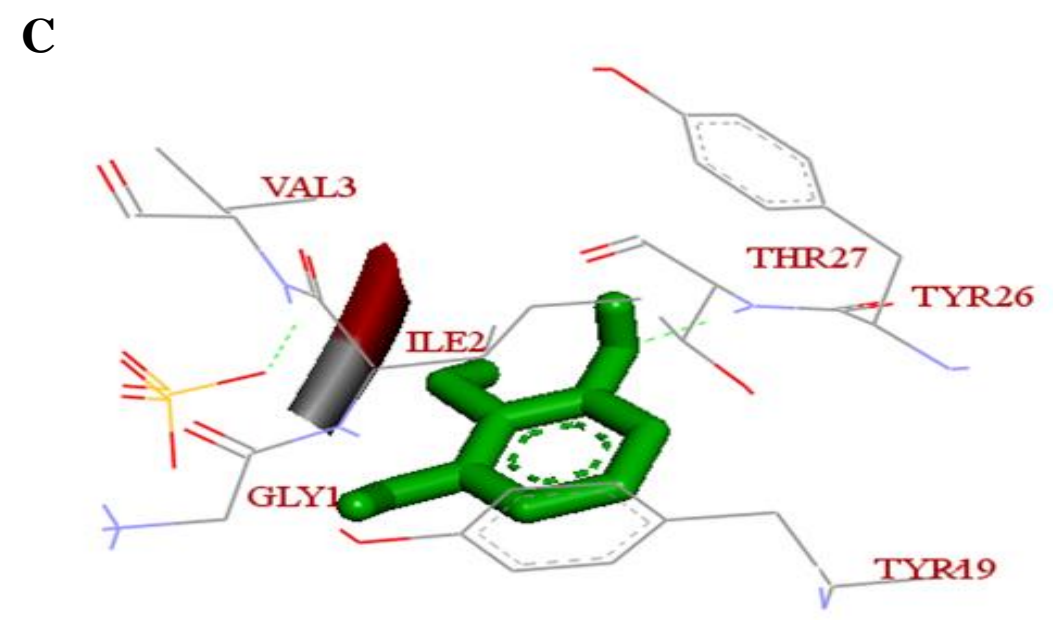

Fig. 5. Evaluation of possible interactions between insulin and pyrogallol using molecular docking. (A), (B) Schematic structure of the binding state of the insulinpyrogallol complex. $(C)$ An accurate view of the state of the insulin-pyrogallol complex.

\section{Discussion}

Targeting protein aggregates by small organic molecules such as polyphenols is one of the most desirable and effective strategies to prevent and improve amyloid disease, which has received much attention in recent years. So far, the anti-amyloidogenic activity of many polyphenols, including quercetin [29], myricetin [30], silibinin [31], tannic acid [32], curcumin [33,34], resveratrol [35], etc. have been investigated. Phenolic compounds in interaction with peptides and proteins, modify their structural properties and prevent amyloid aggregates and their toxicity. In addition, the antioxidant properties of these compounds play an important role in inhibiting amyloid fibrils $[13,14,19]$. It is said that the number of hydroxyl groups and the type of side-chain attached to the aromatic ring in the structure of polyphenols play an important role in the amount of possible binding to proteins so that with increasing the number of hydroxyl groups, the tendency of polyphenols to interact with proteins will be higher [36]. In addition, reports indicate that prevention of $\pi-\pi$ interaction and inhibition of fibrillation process is due to interactions between the phenolic rings of polyphenols and the aromatic residues of amyloidogenic proteins [37]. Among the non-covalent interactions that may occur between polyphenols and proteins, hydrophobic interactions and hydrogen bonds are known as 
the main interactions and are of great importance [36]. The results of molecular docking simulation in this study showed that Py was able to interact with HI through hydrophobic interactions and hydrogen bonding, thereby resulting in the inhibition of HI fibrillation. According to the results, it can be concluded that the presence of an aromatic ring and its attached hydroxyl groups in the Py structure is the reason for the potential of this phenolic compound to interact with the protein.

In this study, $\mathrm{HI}$ protein was exposed to $\mathrm{pH} 2.0$ and a temperature of $50{ }^{\circ} \mathrm{C}$. These conditions lead to the opening of the folded structure of the protein and the formation of amyloid aggregates. The acidic $\mathrm{pH}$ makes the protein highly vulnerable and heat is the main factor in the dissociation of the dimer and the production of monomeric protein [38]. It breaks hydrogen bonds, salt bridges, and disulfide bonds that play an important role in maintaining the native structure of the protein [39] and thus leads to exposure of hydrophobic regions. On the other hand, heating, by increasing the surface hydrophobicity of the protein, provides more binding sites for polyphenol, in which the hydrophobic bonds contribute significantly. It is said that the most interaction between protein and polyphenol is the hydrophobic interactions [40].

Results of our experimental studies (CR absorbance, ThT and ANS fluorescence intensity) showed that Py has significant inhibitory potential against amyloid fibrils formation and is very effective in reducing the exposure of hydrophobic regions and thus reducing the tendency of the protein to form amyloid fibrils. This effect, like the inhibitory effect of many polyphenols, including gallic acid [41], silibinin [42] and curcumin [43] was observed in a dose-dependent manner. In addition, our findings in the study of the effect of Py destabilizing on the process of HI defibrillation indicated the elimination of fibrillar structures and reduction of surface hydrophobicity of protein is due to the disappearance of hydrophobic regions. Also, in this process, with increasing Py concentration, the rate of defibrillation increased. 
The inhibitory effect of Py on other amyloidogenic proteins has also been investigated. According to Di Giovanni et al. [44], Py is effective in preventing the aggregation of $\alpha$-synuclein and inhibiting the conversion of $\beta$-amyloid protofibrils to mature fibrils.

It has been reported that main factor in the inhibitory properties of polyphenols with similar chemical structure is the phenolic hydroxyl group [45]. Our results confirmed this claim. The samples containing $\mathrm{HI}+\mathrm{Ga}(1: 5)$ and the sample containing $\mathrm{HI}+\mathrm{Py}(1: 5)$ showed the same inhibitory effect, so the structural difference between the two compounds (carboxyl group) has no effect on inhibiting the HI fibrillation process. These results support the Jayamani and Shanmugam [41] hypothesis that the carboxyl group is not effective in inhibiting fibril formation. In fact, the trihydroxy benzene group in Ga is the major component of the inhibitory effect in this compound. In a study by Konar et al. [46], the inhibitory effect of several phenolic compounds including benzoic acid (BA), 4-hydroxy benzoic acid (4-HBA), 3,4-dihydroxy benzoic acid (3,4-DHBA), 3,5-dihydroxy benzoic acid (3,5-DHBA), and Py, which their structures are close to the structure of Ga, were investigated on HEWL fibrillation. It was reported that $\mathrm{Ga}$ and Py had the greatest inhibitory effect compared to other compounds. The presence of more hydroxyl groups in $\mathrm{Ga}$ and $\mathrm{Py}$ is the most significant reason for the high inhibitory effect of these two compounds, compared to other phenolic compounds. On the other hand, the similar inhibitory effect of Ga and Py, as well as the ineffectiveness of benzoic acid (which has only one carboxyl group in its structure) in the process of preventing the formation of HEWL fibrils, were other results obtained in this study which led to the conclusion that the carboxyl group had no significant effect on the inhibitory properties of polyphenols.

In another study, the effect of hydroxyl groups of three polyphenolic compounds, myricetin, morin, and flavone, on the elimination of amyloid fibrils was investigated [47]. These three compounds have a similar structure and the only difference is in the number of hydroxyl groups. The results of this study demonstrated that myricetin, with six hydroxyl groups, and morin, with five hydroxyl groups, were more effective at destabilizing amyloid fibrils than flavone, which lacked the 
hydroxyl group. According to Ono et al. [48], the number of hydroxyl groups is directly related to the anti-amyloidogenic effects of polyphenolic compounds.

Investigation of microscopic images of the anti-amyloidogenic effects of small organic molecules on amyloid aggregates in many studies showed that some compounds belonging to this group of inhibitors, in addition to preventing fibril formation, can also redirect amyloidogenic proteins toward the creation of non-fibrillar aggregates. Wang et al. [49] in the study of the effect of Epigallocatechin3-gallate (EGCG) on human insulin fibrillation found that EGCG in physiological conditions, in addition to having high inhibitory effects, leads to a change in the path of aggregation and the formation of globular aggregates. In a study on inhibitory and destabilizing effects of quercetin against bovine insulin fibrillation (at acidic $\mathrm{pH}$ and high temperature) and its defibrillation it was shown that this compound leads to the formation of amorphous aggregates both during inhibition of fibrillation and in the process of elimination of amyloid fibrils [29]. In other studies, the effect of silibinin [42], curcumin [43] and ferulic acid [50] on the formation of bovine insulin fibril also indicated the presence of amorphous species instead of fibrillar morphology in samples containing these compounds. Our observations in this study are consistent with the above reports, as in the microscopic images obtained, the presence of non-fibrillar species and similar to amorphous aggregates in both the inhibitory and destabilizing effects of Py were clearly observed. The results of FTIR spectroscopy also indicate a high probability of amorphous aggregation formation during inhibition and treatment of HI amyloid fibrils by Py.

Totally, based on various studies it can be concluded that polyphenols prevent the formation of amyloid species in different ways. For example, a group of polyphenols, despite being successful in inhibiting the formation of oligomers, lead to the formation of fibrils. Another group prevents the formation of fibrils but has no effect on inhibiting the formation of oligomers. There are also polyphenols that are capable of preventing the formation of both oligomeric and fibrillar forms. Many of them cause a change of the direction of fibrillation and the creation of non-fibrillar oligomers 
instead of fibrillar forms [37]. Regarding these facts, the structure of the compound is very important in determining how it affects the process of amyloid formation.

\section{Materials and methods}

\subsection{Chemicals}

Human insulin (HI), Thioflavin T (ThT), 1-anilino naphthalene 8-sulfonic acid (ANS), Congo red (CR) were purchased from Sigma-Aldrich (St. Louis, MO, USA). Pyrogallol (Py) and all salts and organic solvents were obtained from Merck (Darmstadt, Germany).

\subsection{Preparation of insulin solution for fibril formation}

HI fibrils were prepared according to Amini et al. [51] method. HI (4 mg/ml) was dissolved in a $50 \mathrm{mM}$ glycine $-\mathrm{HCl}$ buffer $(\mathrm{pH} 2.0)$ containing $0.02 \% \mathrm{NaN}_{3}$. Then, it was incubated in the presence and absence of different concentrations of Py (in ratios of $0.1,0.5,1$, and 5 Py to 1 protein in terms of $\mathrm{mM})$ at $50{ }^{\circ} \mathrm{C}$ without agitation.

To investigate the destabilizing effects of Py on $\mathrm{HI}$ amyloid fibrils, preformed fibrils in the presence and absence of different concentrations of Py (in ratios of $0.1,0.5,1$, and 5 Py to 1 protein in terms of $\mathrm{mM}$ ) were incubated at $37^{\circ} \mathrm{C}$ for 3 days.

The formation of HI fibrils was monitored by CR and FTIR spectroscopy, ThT and ANS fluorescence intensity, and atomic force microscopy.

\subsection{Congo red (CR) binding assay}

CR binding assay is mainly used to identify amyloids in vitro. CR was dissolved in potassium phosphate buffer ( $\left.5 \mathrm{mM}, 0.15 \mathrm{M} \mathrm{NaCl}, 0.02 \% \mathrm{NaN}_{3}, \mathrm{pH} 7.4\right)$ and filtered. This stock solution (2 
$\mathrm{mM}$ ) was diluted to a final concentration of $12.5 \mu \mathrm{M}$ and used as a working solution. $5 \mu \mathrm{l}$ of each sample solution was added to $195 \mu \mathrm{l}$ of CR working solution and incubated at room temperature and in the dark for 30 minutes. The spectrum of CR was measured at a wavelength of 400-700 nm using an Epoch microplate reader (BioTek, USA).

\subsection{Thioflavin $T(T h T)$ fluorescence assay}

ThT fluorescence was also used as a reliable method in identifying HI amyloid fibrils and also evaluating the effect of Py on HI fibrillation. After binding of ThT to fibrillar structures, dye fluorescence intensity significantly increases [52].

ThT was dissolved in sodium phosphate buffer $\left(10 \mathrm{mM}, 0.15 \mathrm{M} \mathrm{NaCl}, 0.02 \% \mathrm{NaN}_{3}, \mathrm{pH} 7.0\right)$ and filtered. This stock solution $(2 \mathrm{mM})$ was diluted to a final concentration of $20 \mu \mathrm{M}$ and used as a working solution. $15 \mu \mathrm{l}$ of each sample solution was added to $585 \mu \mathrm{l}$ of ThT working solution and incubated at room temperature for 2 minutes. Then, the ThT fluorescence intensity was measured with excitation at $440 \mathrm{~nm}$ and emission at $488 \mathrm{~nm}$ and the slit widths for excitation and emission 5 $\mathrm{nm}$ and $10 \mathrm{~nm}$, respectively, using the Cary-Eclipse fluorescence spectrophotometer (Agilent, USA).

To obtain the sigmoid curve, the data collected from ThT fluorescence measurements at $485 \mathrm{~nm}$ were fitted by the following equation [53]:

$$
\boldsymbol{F}=\frac{F_{\max }}{1+e^{-\left(\left[\frac{t-t_{0}}{\tau}\right]\right)}}
$$

Where $\mathrm{F}$ is the fluorescence intensity at time $\mathrm{t}, \mathrm{t}$ is the incubation time, $\mathrm{F}_{\max }$ is the fluorescence intensity at the final time and $t_{0}$ is the time to reach $50 \%$ of the maximum fluorescence. The value of $\tau$ (the time constant of fibril growth) was obtained by nonlinear regression. Kapp (the apparent growth rate constant of fibril) and lag phase time were obtained from $1 / \tau$ and $t_{0}-2 \tau$, respectively.

The inhibition percentage was calculated by the following equation [54]: 


$$
\text { Inhibition percentage }=\frac{I_{\text {control }}-I}{I_{\text {control }}} \times 100
$$

Where I is the final ThT fluorescence intensity.

\subsection{Anilinonaphthalene-8-sulfonic acid (ANS) fluorescence assay}

ANS is generally used to identify partially folded intermediates and to investigate the surface hydrophobicity of proteins. The fluorescence intensity of this dye is significantly increased due to its binding to hydrophobic parts in proteins [55]. Therefore, ANS fluorescence is a very suitable method to identify amyloid structures due to their high surface hydrophobicity.

ANS was dissolved in sodium phosphate buffer (10 mM, $\left.0.15 \mathrm{M} \mathrm{NaCl}, 0.02 \% \mathrm{NaN}_{3}, \mathrm{pH} 7.0\right)$ and filtered. This stock solution $(2 \mathrm{mM})$ was diluted to a final concentration of $20 \mu \mathrm{M}$ and used as a working solution. $15 \mu \mathrm{l}$ of each sample solution was added to $585 \mu \mathrm{l}$ of ANS working solution, mixed, and incubated at room temperature for 1 minute. The intensity of ANS fluorescence was measured with excitation at $380 \mathrm{~nm}$ and emission at 420-600 $\mathrm{nm}$ and the slit widths for excitation and emission $5 \mathrm{~nm}$ and $10 \mathrm{~nm}$, respectively, using the Cary-Eclipse fluorescence spectrophotometer (Agilent, USA).

\subsection{Fourier transform infrared (FTIR) spectroscopic assay}

To investigate changes in the secondary structure of the protein, according to Kawasaki et al. [56] method, Protein samples were mixed with completely dry $\mathrm{KBr}$ and compressed into a thin disc using a tablet press. Then all spectra were recorded from $400 \mathrm{~cm}^{-1}$ to $4000 \mathrm{~cm}^{-1}$ at a resolution of $4 \mathrm{~cm}^{-1}$ with 16 scans and by the 8400 S spectrometer. (Shimadzu, Japan).

\subsection{Atomic force microscopy (AFM)}


To investigate the effects of Py on the morphology of HI amyloid fibrils, $5 \mu$ of each sample diluted with deionized water was dried on a mica plate in the presence of air. After drying, the samples were imaged in the non-contact AFM (NC-AFM) imaging mode at a scan frequency of $0.5 \mathrm{~Hz}$ using Full Plus AFM (Ara Pajoohesh, Iran).

\subsection{Docking studies}

For molecular docking analysis, the structure of HI was obtained from the protein data bank (http://rcsb.org/) (PDB id code: 1GUJ) and the SDF file of Py was obtained from PubChem database (http://pubchem.ncbi.nlm.nih.gov/) (PubChem CID: 2519). Protein docking was performed using Autodock v1.5.6 software. Discovery studio and VMD v1.9.3 program software were also used to prepare the $2 \mathrm{D}$ and $3 \mathrm{D}$ schematic diagrams of the docking model, as well as to exhibit different orientations between ligand and the protein.

\subsection{Statistical analysis}

Results were presented as mean \pm standard deviation (SD). All experiments were performed at least three times and one-way analysis of variance (ANOVA) was used. P-value $<0.05$ was considered significant. GraphPad Prism software version 9.2.0 was used for statistical analysis.

\section{Conclusion}

In this study, it was found that Py has a very acceptable function both as an inhibitor in preventing the formation of amyloid fibrils and as a destabilizer in eliminating pre-formed amyloid fibrils. The phenolic structure of Py seems to play an important role in creating effective interactions with the protein. Since various studies have shown that natural polyphenols have special properties to prevent 
amyloid disease, the present study could be useful in advancing the design purposes of new antiamyloid drugs in the future. In addition, more studies are needed to investigate the toxicity of Py in cellular conditions and then to investigate the anti-amyloidogenic properties of Py in animal models in order to prove its medicinal potential.

Author Contributions: Perform experiments, analyze data, write the paper, P.S.; project designer, Editing, Supervision, S.B.; project adviser, S.H.H.

Funding: This study was supported by Lorestan University (Lorestan, Iran).

Conflicts of Interest: The authors declare no conflict of interest.

\section{References}

1. Chiti, F.; Dobson, C.M. Protein misfolding, functional amyloid, and human disease. Annu. Rev. Biochem. 2006, 75, 333-366.

2. Invernizzi, G.; Papaleo, E.; Sabate, R.; Ventura, S. Protein aggregation: Mechanisms and functional consequences. Int. J. Biochem. Cell Biol. 2012, 44, 1541-1554.

3. Knowles, T.P.J.; Vendruscolo, M.; Dobson, C.M. The amyloid state and its association with protein misfolding diseases. Nat. Rev. Mol. Cell Biol. 2014, 15, 384-396.

4. Rochet, J.C. Novel therapeutic strategies for the treatment of protein-misfolding diseases. Expert Rev. Mol. Med. 2007, 9, 1-34.

5. Cheng, B.; Gong, H.; Xiao, H.; Petersen, R.B.; Zheng, L.; Huang, K. Inhibiting toxic aggregation of amyloidogenic proteins: A therapeutic strategy for protein misfolding diseases. Biochim. Biophys. Acta - Gen. Subj. 2013, 1830, 4860-4871. 
6. Hua, Q.X.; Weiss, M.A. Mechanism of insulin fibrillation: The structure of insulin under amyloidogenic conditions resembles a protein-folding intermediate. J. Biol. Chem. 2004, 279, 21449-21460, doi:10.1074/jbc.M314141200.

7. Groenning, M.; Frokjaer, S.; Vestergaard, B. Formation Mechanism of Insulin Fibrils and Structural Aspects of the Insulin Fibrillation Process. Curr. Protein Pept. Sci. 2009, 10, 509528, doi:10.2174/138920309789352038.

8. Okamura, S.; Hayashino, Y.; Kore-Eda, S.; Tsujii, S. Localized amyloidosis at the site of repeated insulin injection in a patient with type 2 diabetes. Diabetes Care 2013, 36.

9. Shikama, Y.; Kitazawa, J.I.; Yagihashi, N.; Uehara, O.; Murata, Y.; Yajima, N.; Wada, R.; Yagihashi, S. Localized amyloidosis at the site of repeated insulin injection in a diabetic patient. Intern. Med. 2010, 49, 397-401, doi:10.2169/internalmedicine.49.2633.

10. Ahmad, A.; Millett, I.S.; Doniach, S.; Uversky, V.N.; Fink, A.L. Partially folded intermediates in insulin fibrillation. Biochemistry 2003, 42, 11404-11416, doi:10.1021/bi034868o.

11. Tartaglia, G.G.; Pawar, A.P.; Campioni, S.; Dobson, C.M.; Chiti, F.; Vendruscolo, M. Prediction of Aggregation-Prone Regions in Structured Proteins. J. Mol. Biol. 2008, 380, 425436, doi:10.1016/j.jmb.2008.05.013.

12. Selivanova, O.M.; Galzitskaya, O. V. Structural polymorphism and possible pathways of amyloid fibril formation on the example of insulin protein. Biochem. 2012, 77, 1237-1247.

13. Sgarbossa, A. Natural biomolecules and protein aggregation: Emerging strategies against amyloidogenesis. Int. J. Mol. Sci. 2012, 13, 17121-17137.

14. Porat, Y.; Abramowitz, A.; Gazit, E. Inhibition of amyloid fibril formation by polyphenols: Structural similarity and aromatic interactions as a common inhibition mechanism. Chem. Biol. Drug Des. 2006, 67, 27-37. 
15. Žerovnik, E. Amyloid-fibril formation: Proposed mechanisms and relevance to conformational disease. Eur. J. Biochem. 2002, 269, 3362-3371.

16. Shaham-Niv, S.; Rehak, P.; Zaguri, D.; Levin, A.; Adler-Abramovich, L.; Vuković, L.; Král, P.; Gazit, E. Differential inhibition of metabolite amyloid formation by generic fibrillationmodifying polyphenols. Commun. Chem. 2018, 1, doi:10.1038/s42004-018-0025-z.

17. Ozcan, T.; Akpinar-Bayizit, A.; Yilmaz-Ersan, L.; Delikanli, B. Phenolics in Human Health. Int. J. Chem. Eng. Appl. 2014, 5, 393-396, doi:10.7763/ijcea.2014.v5.416.

18. Pandey, K.B.; Rizvi, S.I. Plant polyphenols as dietary antioxidants in human health and disease. Oxid. Med. Cell. Longev. 2009, 2, 270-278.

19. Stefani, M.; Rigacci, S. Protein folding and aggregation into amyloid: The interference by natural phenolic compounds. Int. J. Mol. Sci. 2013, 14, 12411-12457.

20. Ozturk Sarikaya, S.B. Acethylcholinesterase inhibitory potential and antioxidant properties of pyrogallol. J. Enzyme Inhib. Med. Chem. 2015, 30, 761-766, doi:10.3109/14756366.2014.965700.

21. Shin, M.; Park, E.; Lee, H. Plant-Inspired Pyrogallol-Containing Functional Materials. Adv. Funct. Mater. 2019, 29.

22. Alavi Rafiee, S.; Farhoosh, R.; Sharif, A. Antioxidant Activity of Gallic Acid as Affected by an Extra Carboxyl Group than Pyrogallol in Various Oxidative Environments. Eur. J. Lipid Sci. Technol. 2018, 120, doi:10.1002/ejlt.201800319.

23. Nielsen, L.; Khurana, R.; Coats, A.; Frokjaer, S.; Brange, J.; Vyas, S.; Uversky, V.N.; Fink, A.L. Effect of environmental factors on the kinetics of insulin fibril formation: Elucidation of the molecular mechanism. Biochemistry 2001, 40, 6036-6046, doi:10.1021/bi002555c. 
24. Shivu, B.; Seshadri, S.; Li, J.; Oberg, K.A.; Uversky, V.N.; Fink, A.L. Distinct $\beta$-sheet structure in protein aggregates determined by ATR-FTIR spectroscopy. Biochemistry 2013, 52, doi:10.1021/bi400625v.

25. Das, S.; Bhattacharyya, D. Destabilization of Human Insulin Fibrils by Peptides of Fruit Bromelain Derived From Ananas comosus (Pineapple). J. Cell. Biochem. 2017, 118, 48814896, doi:10.1002/jcb.26173.

26. Sawaya, M.R.; Sambashivan, S.; Nelson, R.; Ivanova, M.I.; Sievers, S.A.; Apostol, M.I.; Thompson, M.J.; Balbirnie, M.; Wiltzius, J.J.W.; McFarlane, H.T.; et al. Atomic structures of amyloid cross- $\beta$ spines reveal varied steric zippers. Nature 2007, 447, 453-457, doi:10.1038/nature05695.

27. Alam, P.; Beg, A.Z.; Siddiqi, M.K.; Chaturvedi, S.K.; Rajpoot, R.K.; Ajmal, M.R.; Zaman, M.; Abdelhameed, A.S.; Khan, R.H. Ascorbic acid inhibits human insulin aggregation and protects against amyloid induced cytotoxicity. Arch. Biochem. Biophys. 2017, 621, 54-62, doi:10.1016/j.abb.2017.04.005.

28. Siddiqi, M.K.; Alam, P.; Iqbal, T.; Majid, N.; Malik, S.; Nusrat, S.; Alam, A.; Ajmal, M.R.; Uversky, V.N.; Khan, R.H. Elucidating the inhibitory potential of designed peptides against amyloid fibrillation and amyloid associated cytotoxicity. Front. Chem. 2018, 6, doi:10.3389/fchem.2018.00311.

29. Wang, J.B.; Wang, Y.M.; Zeng, C.M. Quercetin inhibits amyloid fibrillation of bovine insulin and destabilizes preformed fibrils. Biochem. Biophys. Res. Commun. 2011, 415, 675-679, doi:10.1016/j.bbrc.2011.10.135.

30. Zelus, C.; Fox, A.; Calciano, A.; Faridian, B.S.; Nogaj, L.A.; Moffet, D.A. Myricetin Inhibits Islet Amyloid Polypeptide (IAPP) Aggregation and Rescues Living Mammalian Cells from IAPP Toxicity. Open Biochem. J. 2012, 6, 66-70, doi:10.2174/1874091x01206010066. 
31. Cheng, B.; Gong, H.; Li, X.; Sun, Y.; Zhang, X.; Chen, H.; Liu, X.; Zheng, L.; Huang, K. Silibinin inhibits the toxic aggregation of human islet amyloid polypeptide. Biochem. Biophys. Res. Commun. 2012, 419, 495-499, doi:10.1016/j.bbrc.2012.02.042.

32. Ono, K.; Hasegawa, K.; Naiki, H.; Yamada, M. Anti-amyloidogenic activity of tannic acid and its activity to destabilize Alzheimer's $\beta$-amyloid fibrils in vitro. Biochim. Biophys. Acta Mol. Basis Dis. 2004, 1690, 193-202, doi:10.1016/j.bbadis.2004.06.008.

33. Ono, K.; Hasegawa, K.; Naiki, H.; Yamada, M. Curcumin Has Potent Anti-Amyloidogenic Effects for Alzheimer's $\beta$-Amyloid Fibrils In Vitro. J. Neurosci. Res. 2004, 75, 742-750, doi:10.1002/jnr.20025.

34. Pandey, N.; Strider, J.; Nolan, W.C.; Yan, S.X.; Galvin, J.E. Curcumin inhibits aggregation of $\alpha$-synuclein. Acta Neuropathol. 2008, 115, 479-489, doi:10.1007/s00401-007-0332-4.

35. Feng, Y.; Wang, X. ping; Yang, S. gao; Wang, Y. jiong; Zhang, X.; Du, X. ting; Sun, X. xia; Zhao, M.; Huang, L.; Liu, R. tian Resveratrol inhibits beta-amyloid oligomeric cytotoxicity but does not prevent oligomer formation. Neurotoxicology 2009, 30, 986-995, doi:10.1016/j.neuro.2009.08.013.

36. Ozdal, T.; Yalcinkaya, İ.E.; Toydemir, G.; Capanoglu, E. Polyphenol-protein interactions and changes in functional properties and digestibility. In Encyclopedia of Food Chemistry; 2018; pp. 566-577 ISBN 9780128140451.

37. Ngoungoure, V.L.N.; Schluesener, J.; Moundipa, P.F.; Schluesener, H. Natural polyphenols binding to amyloid: A broad class of compounds to treat different human amyloid diseases. Mol. Nutr. Food Res. 2015, 59, 8-20.

38. Whittingham, J.L.; Scott, D.J.; Chance, K.; Wilson, A.; Finch, J.; Brange, J.; Guy Dodson, G. Insulin at $\mathrm{pH}$ 2: Structural analysis of the conditions promoting insulin fibre formation. $J$. Mol. Biol. 2002, 318, 479-490, doi:10.1016/S0022-2836(02)00021-9. 
39. Arora, A.; Ha, C.; Park, C.B. Insulin amyloid fibrillation at above $100^{\circ} \mathrm{C}$ : New insights into protein folding under extreme temperatures. Protein Sci. 2004, 13, 2429-2436, doi:10.1110/ps.04823504.

40. Siebert, K.J.; Troukhanova, N. V.; Lynn, P.Y. Nature of Polyphenol-Protein Interactions. J. Agric. Food Chem. 1996, 44, 80-85, doi:10.1021/jf9502459.

41. Jayamani, J.; Shanmugam, G. Gallic acid, one of the components in many plant tissues, is a potential inhibitor for insulin amyloid fibril formation. Eur. J. Med. Chem. 2014, 85, 352358, doi:10.1016/j.ejmech.2014.07.111.

42. Katebi, B.; Mahdavimehr, M.; Meratan, A.A.; Ghasemi, A.; Nemat-Gorgani, M. Protective effects of silibinin on insulin amyloid fibrillation, cytotoxicity and mitochondrial membrane damage. Arch. Biochem. Biophys. 2018, 659, 22-32, doi:10.1016/j.abb.2018.09.024.

43. Rabiee, A.; Ebrahim-Habibi, A.; Ghasemi, A.; Nemat-Gorgani, M. How curcumin affords effective protection against amyloid fibrillation in insulin. Food Funct. 2013, 4, 1474-1480, doi:10.1039/c3fo00019b.

44. Di Giovanni, S.; Eleuteri, S.; Paleologou, K.E.; Yin, G.; Zweckstetter, M.; Carrupt, P.A.; Lashuel, H.A. Entacapone and tolcapone, two catechol O-methyltransferase inhibitors, block fibril formation of $\alpha$-synuclein and $\beta$-amyloid and protect against amyloid-induced toxicity. J. Biol. Chem. 2010, 285, 14941-14954, doi:10.1074/jbc.M109.080390.

45. Liu, Y.; Pukala, T.L.; Musgrave, I.F.; Williams, D.M.; Dehle, F.C.; Carver, J.A. Gallic acid is the major component of grape seed extract that inhibits amyloid fibril formation. Bioorganic Med. Chem. Lett. 2013, 23, 6336-6340, doi:10.1016/j.bmcl.2013.09.071.

46. Konar, M.; Ghosh, D.; Roy, P.; Dasgupta, S. Probing the role of ortho-dihydroxy groups on lysozyme fibrillation. Int. J. Biol. Macromol. 2018, 109, 619-628, doi:10.1016/j.ijbiomac.2017.12.115. 
47. Gargari, S.A.; Barzegar, A.; Tarinejad, A. The role of phenolic OH groups of flavonoid compounds with H-bond formation ability to suppress amyloid mature fibrils by destabilizing $\beta$-sheet conformation of monomeric A $\beta 17-42$. PLoS One 2018, 13, doi:10.1371/journal.pone.0199541.

48. Ono, K.; Hamaguchi, T.; Naiki, H.; Yamada, M. Anti-amyloidogenic effects of antioxidants: Implications for the prevention and therapeutics of Alzheimer's disease. Biochim. Biophys. Acta - Mol. Basis Dis. 2006, 1762, 575-586.

49. Wang, S.H.; Dong, X.Y.; Sun, Y. Effect of (-)-epigallocatechin-3-gallate on human insulin fibrillation/aggregation kinetics. Biochem. Eng. J. 2012, 63, 38-49, doi:10.1016/j.bej.2012.02.002.

50. Jayamani, J.; Shanmugam, G.; Azhagiya Singam, E.R. Inhibition of insulin amyloid fibril formation by ferulic acid, a natural compound found in many vegetables and fruits. RSC Adv. 2014, 4, 62326-62336, doi:10.1039/c4ra11291a.

51. Amini, R.; Yazdanparast, R.; Bahramikia, S. Apigenin reduces human insulin fibrillation in vitro and protects SK-N-MC cells against insulin amyloids. Int. J. Biol. Macromol. 2013, 60, 334-340, doi:10.1016/j.ijbiomac.2013.06.013.

52. Biancalana, M.; Koide, S. Molecular mechanism of Thioflavin-T binding to amyloid fibrils. Biochim. Biophys. Acta - Proteins Proteomics 2010, 1804, 1405-1412.

53. Esmaeili, S.; Ghobadi, N.; Akbari, V.; Moradi, S.; Shahlaie, M.; Ghobadi, S.; Jalalvand, A.R.; Amani, M.; Khodarahmi, R. Pyridine-2,3-dicarboxylate, quinolinic acid, induces 1N4R Tau amyloid aggregation in vitro: Another evidence for the detrimental effect of the inescapable endogenous neurotoxin. Chem. Biol. Interact. 2020, 315, 108884, doi:10.1016/j.cbi.2019.108884. 
54. Phan, H.T.T.; Samarat, K.; Takamur, Y.; Azo-Oussou, A.F.; Nakazono, Y.; Vestergaard, M.C. Polyphenols modulate alzheimer's amyloid beta aggregation in a structure-dependent manner. Nutrients 2019, 11, doi:10.3390/nu11040756.

55. Sulatsky, M.I.; Sulatskaya, A.I.; Povarova, O.I.; Antifeeva, I.A.; Kuznetsova, I.M.; Turoverov, K.K. Effect of the fluorescent probes ThT and ANS on the mature amyloid fibrils. Prion 2020, 14, 67-75, doi:10.1080/19336896.2020.1720487.

56. Kawasaki, T.; Fujioka, J.; Imai, T.; Torigoe, K.; Tsukiyama, K. Mid-infrared free-electron laser tuned to the amide I band for converting insoluble amyloid-like protein fibrils into the soluble monomeric form. Lasers Med. Sci. 2014, 29, doi:10.1007/s10103-014-1577-5. 